\title{
Urgences
}

\section{Mots de passe}

\section{Élisabeth Haghebaert}

Numéro 26, décembre 1989

Des textes qui chantent

URI : https://id.erudit.org/iderudit/025551ar

DOI : https://doi.org/10.7202/025551ar

Aller au sommaire du numéro

Éditeur(s)

Urgences

ISSN

0226-9554 (imprimé)

1927-3924 (numérique)

Découvrir la revue

Citer cet article

Haghebaert, É. (1989). Mots de passe. Urgences, (26), 14-27.

https://doi.org/10.7202/025551ar

Ce document est protégé par la loi sur le droit d'auteur. L'utilisation des services d'Érudit (y compris la reproduction) est assujettie à sa politique d'utilisation que vous pouvez consulter en ligne.

https://apropos.erudit.org/fr/usagers/politique-dutilisation/
Cet article est diffusé et préservé par Érudit.

Érudit est un consortium interuniversitaire sans but lucratif composé de l’Université de Montréal, l'Université Laval et l'Université du Québec à Montréal. Il a pour mission la promotion et la valorisation de la recherche. https://www.erudit.org/fr/ 


\section{Mots de passe Élisabeth Haghebaert}

Les yeux réduits à des fentes et pris dans les orbites comme dans des lunettes aux bords taillés. "Au revoir, mon Chinois de la douleur ", dit l'ami.

\section{Peter Handke}

le chinois

met l'œuvre

à la place du miroir

Marie Redonnet

\section{Le Chinols ${ }^{1}$}

I

Un Chinois reste dans ma rue

Vieux Chinois qui courbe l'échine

On ne l'a pas très souvent vu

Car tout autour de sa maison

5 II y a un mur de Chine

II

Ce Chinois c'est un Robinson

Sa maison c'est son île

Il vit il meurt à sa façon

A l'abri des p'tits polissons

10 Qui n'sav'nt mêm' pas c'que c'est qu'la Chine

III

Chinois tu courbes le dos

Quel est donc ton fardeau

1 Paroles et musique de Georges Dor, arrangements de François Cousineau. Chanson écrite en 1966 et enregistrée en avril 1968, d'après les renseignements fournis par l'auteur lors d'une aimable conversation téléphonique, le 24 aout 1989. La chanson se trouve donc sur le deuxième disque de Georges Dor - disque Gamma GM-GS 113 - intitule Mes ormes dans la plaine, qui saurait parler de vous?, arrangements musicaux de François Cousineau, "dans le sens de ce qu'un Occidental perçoit de la musique orientale ". Le texte figure dans le premier des quatre recueils intitulés Poémes et chansons, 1, 2, 3 et 4, Montréal, Leméac/ L'Hexagone, publies respectivement en 1968, 1971, 1972 et 1980, p. 51-52. 
Est-ce l'exil que tu portes

Sur ton dos...

IV

15 Soir et matin mon vieux Chinois

Quand il fait sa prière.

Est-c' que monsieur Bouddha l'entend

Du plus profond de l'Orient

Perdu dans ses chimères

V

20 La vie là-bas a bien changé

Mais la Chine est la même

Millénaire et désenchantée

Inaccessible et tourmentée

Derrière sa muraille

VI

25 Vieux Chinois tu te souviens

De Nankin de Pékin

De Canton de Sou-Tchéou

De chez vous...

VII

Moi je m'souviens des p'tits Chinois

30 A vingt-cinq sous la pièce

Étaient-ce tes petits enfants

Que j'achetais à ce prix-là

Le cœur plein d'allégresse

VIII

Mais que sont-ils donc devenus

35 Dans leur terre lointaine

Ces enfants qui marchaient pieds nus

La longue marche du salut

Avec Mao en tête

IX

Vieux Mao je te salue

40 Un Chinois dans ma rue

T'envoie le bonjour Mao

Et s'incline...

$X$

Je n'aurai jamais vu la Chine

Mais je me l'imagine

45 Dans les vers de Mao Tsé-Toung 
Hommes et femm's arbres et fleurs

A travers brum's et bruine

$X I$

Quant au Chinois moi j'ai le mien

Dans ma rue bien tranquille

50 Un Chinois qui va et qui vient

A petits pas soir et matin

Comme on le fait en Chine

XII

Mais il reste l'étranger

Dans un décor empesé

55 Les yeux mi-clos pour mieux rêver

A la Chine...

\section{Envoi}

Trente ans plus tard, on n'achète plus les "p'tits Chinois " mais la Chine se vend bien encore ${ }^{2}$ et, à la puissance de fascination de la métaphore des lointains mystérieux, se superpose la puissance de l'actualité, cyclique comme un refrain monocorde.

\section{Place de la chanson, 1}

"Cest [...] dans les rues que courent les chansons. » ${ }^{3}$ Dans les rues tranquilles où se font les révolutions. Pièce de circonstance ${ }^{4}$ ou morceau d'idéologie affective née du décor urbain, cette chanson pour un passant - tel vieux Chinois de Longueuil - écrite dans le contexte de la Révolution culturelle chinoise de 1966, se classe au répertoire des chansons éphémères peu connues ${ }^{5}$, victime de l' «insoutenable légèreté » du genre.

2 Ne citons que le numéro 55-56 (3० trimestre1987) de la revue Dérives: a Impressions de Chine " et le numéro $507-508$ (aout 1989) de la revue Critique: * Chine 1949-1989".

3 Georges Dor, Si tu savais, essai autobiographique, Montréal, Éditions de l'Homme, 1977, p. 30.

4 Confirmé par l'auteur: \& Il y avait bien un vieux Chinois dans ma rue, ou je devrais dire: j'habitais dans la rue du Chinois. La maison a été détruite depuis ." Rappelons egalement qu'à cette époque Georges Dor travaillait au Téléjournal de Radio-Canada, ibid., p. 42.

5 Exception faite de l'étude collective de 1968 justement, date d'enregistrement du disque, par le Département d'études canadiennes de l'Université Laval 
On sait que les textes des chansonniers résistent mal à la lecture et passent vite en raison de leur apparente pauvreté parolière ${ }^{6}$. Cet état de fait n'a pas empêché pas Georges Dor de prendre le parti de la chanson. Éthique personnelle au service du quotidien et du populaire:

La chanson a la vocation qui lui revient, qui n'est pas celle de vouloir remplacer la poésie, la littérature ou la musique, mais simplement d'être elle-même: quotidienne, accompagnatrice du quotidien, révélatrice aussi de ce quotidien, avec, de temps à autre, l'heureux hasard qui fait de l'une d'entre elles le témoin d'une époque. 7

C'est ainsi que du «Chinois" de Dor on retient en premier lieu l'orientalisme musical de bazar, héritage de l'époque coloniale, véhiculé par les missionnaires et les films américains: une idée ready-made, entre factuel descriptif et rêverie exotisante, ponctuée de coups de cymbales et de gong.

Dans cette perspective, l'insignifiance apparente de l'objet de consommation que constitue la chanson ne peut que fixer l'attention sur son in-signifiance ${ }^{8}$ comme objet clos : ce qui de ce non-poème ${ }^{9}$ délibéré ou de cette poésie du quotidien fait un texte appelant une mélodie ${ }^{10}$ pour l'illustrer, le souligner

sur le thème de la * Chanson d'hier et d'àujourd'hui ", qui, dans la partie consacrée à Georges Dor (p. 98-131), mentionne Le Chinois.

6 Pour une mise en perspective générale de la chanson québécoise, voir le livre de Bruno Roy, Et cette Amérique chante en québécois, coll. "Les beauxarts ", Montréal, Leméac, 1978, 295 p.

7 Si tu savais, p. 30 . A propos du traditionnel débat sur la place à accorder a la chanson - qualifié de a genre mineur " par certains -, qu'il suffise de rappeler qu'en 1962, les Éditions Seghers accueillent un Léo Ferré dans la collection « Poètes d'aujourdhui " ( $n^{\circ} 93$ ) et qu'en 1963, avec le Georges Brassens ( $n^{\circ} 99$ ), apparait la mention a Poesie et chansons » qui, de soussection, deviendra, au tournant des annees 1970, la collection "Chansons d'aujourd'hui ". On note par exemple, en effet, dans la a chansonnographie" consacrée a Aragon (1967), que: * La chanson est un des plus hauts moyens d'expression français ", ou l'amorce d'une reconnaissance de fait.

8 Au sens de signification intériorisée.

9 À distinguer de la notion de a non-poeme "selon Gaston Miron: « Le nonpoème c'est ma tristesse ontologique / La souffrance d'être un autre. "Gaston Miron: a Notes sur le non-poeme et le poeme " [1965], in L'homme rapaille, coll. "Prix de la revue Etudes françaises". Montréal, Presses de l'Université de Montréal, 1970, p. 122. Ici, ce terme est employé comme lieu intermédiaire entre poème au sens littéraire et texte de chanson.

10 Interrogé sur ses méthodes de production, Georges Dor confirme que, n'étant pas musicien, une fois le texte écrit, il le laisse de cóté, ж en attendant 
18

et le ponctuer recto tono. Ou comment, en quelques mots à fredonner, le travail du texte vient doubler, miroir magique, sur le plan phonique et sémantique, souterrainement ou infratextuellement, telle thématique à coloration socio-culturelle et créer un état de résonance émotive. Avant d'aborder le texte dans sa textualité donc, les remarques structurelles d'usage, et quelques commentaires sur l'originalité du paradigme structurant en regard du contexte.

\section{Place à la chanson: le texte chiffré}

Au poème traditionnel, le texte de la chanson emprunte une forme fixe qui n'est qu'apparente. L'orthodoxie de cette forme, constituée d'un appareil de rimes mêlées à prédominance masculine, est en effet contestable. L'intérêt de son organisation, irréductible à un modèle simple - de type a-b-ab ou a-b-b-a, par exemple -, consiste, semble-t-il, à contrebalancer la rigueur de la structure compositionnelle d'ensemble: un total de cinquante-six vers répartis en douze strophes comme suit: huit quintils et quatre quatrains hétérométriques. Les groupes de quintils ainsi constitués (strophes III, IV-V, VII-VIII, X-XI) sont composés exclusivement de vers octo- et hexasyllabiques tandis que les quatrains, recourant aux vers hexa- et octosyllabiques pour les deux premiers et aux vers hexa-, hepta- et octosyllabiques pour les deux derniers, ont pour point commun de se terminer par un vers trisyllabique. Les strophes III, VI, IX, XII découpent ainsi le texte en quatre groupes de deux quintils donnant deux fois quatre blocs (quatre de deux strophes et quatre d'une strophe en alternance) distribués suivant un algorythme de 2,1, 2,1, 2,1, 2,1. La chanson se trouve de la sorte structurée sous le signe d'une parité que sous-tend le rythme à quatre temps de la mélodie. Parité du chansonnier et du personnage ou d'une autre réalité qui convoquerait d'un côté la Chine, de l'autre le Québec? Ceci relève de l'hypothèse interprétative et de l'indécidable.

L'examen syntaxique révèle cependant qu'une structure se dessine également au niveau de l'emploi des pronoms. La narration descriptive au «je» et au «il» qui se manifeste dans les quintils cède en effet la place au discours phatique et

qu'il chante "; $\infty$ que mentionnait déjà Bruno Roy. Et cette Amérique chante en québécois, Montréal, Leméac, 1978, p. 40. 
s'adresse au «tu»dans trois des quatre quatrains qui s'(in)achèvent tous sur un point de suspension: discours dialogique à sens unique. À la place de l'«autre», personnage enfermé dans son "étrangéité»"11, répond le silence. Sans doute la structure paradigmatique pourra-t-elle apporter quelque élément susceptible d'étayer ces premiers signes extérieurs de sens, mais encore faut-il, compte tenu de l'éloignement temporel, replacer le texte en contexte.

\section{Place de la chanson, 2}

Certes, de nos jours, le métissage des cultures a fait de l'étranger ou de l'exilé un objet de curiosité banal ${ }^{12}$. On sait aussi maintenant que ces thèmes sont des constantes littéraires particulièrement présentes dans le corpus québécois ${ }^{13}$. Simplement, la notion d' "étranger" s'est-elle modifiée, élargie à la taille planétaire. L'« étranger au village" apporte maintenant avec lui une dimension transculturelle. Comme le note Pierre Nepveu:

L'imaginaire québécois [...] s'est largement défini, depuis les années soixante, sous le signe de l'exil [...], du manque, du pays absent ou inachevé et, du milieu même de cette négativité, s'est constitué en imaginaire migrant, pluriel, souvent cosmopolite. ${ }^{14}$

Il reste que dans le cadre des jeunes années 1960, parler de l'étranger comme tel et d'un Chinois en particulier est chose rare, voire exceptionnelle en chanson ${ }^{15}$ au

11 Proposition de néologisme pour désigner l'étrangeté même d'être étranger et de se voir être vu comme tel: une étrangeté à la puissance deux.

12 Il suffit de penser, récemment, à Chinatown de Daniel Lavoie et, phénomène encore plus prégnant en France, aux chansons de Renaud (Deuxième génération, par exemple) ou de Charlélie Couture. Signe des temps et de l'émergence des minorités visibles. Interrogé sur ce point, Georges Dor a répondu que, s'il avait à refaire cette chanson, il parlerait non seulement de son Chinois mais des diverses communautés ethniques de Montréal.

13 Qu'on pense seulement au Survenant de Germaine Guèvremont (1945), par exemple.

14 Pierre Nepveu, L'écologie du réel, coll. « Papiers collés ", Montréal, Boréal, 1988, p. 201.

15 Exception faite de La petite Tonkinoise (1906, paroles de Villard-Christiné, musique de Vincent Scotto, d'après les informations gracieusement fournies par le personnel de la discothèque de Radio-Canada) interprétée par Maurice Chevalier, fortement teintée de colonialisme, on citera à titre de comparaison 
Québec ${ }^{16}$ : c'est - osons le mauvais jeu de mots — « du chinois ». Toute lacune méritant interrogation, on peut alléguer au sujet de cette absence que la tendance allait alors dans le sens de l'enracinement et s'attachait en priorité à la notion du pays.

Georges Dor ne fait pas autre chose qu'en parler, du pays, mais à rebours, par défaut, de façon détournée - allant jusqu'à maudire le *Maudit pays* ${ }^{17}$. S'il évoque ici le pays manqué, l'Orient mythique, qui fera dire à Charlebois: « Cartier, Cartier, si t’avais navigué du bon côté » ${ }^{18}$, c'est par référence à l'expérience quotidienne à la fois oblitérante et révélatrice de l'ailleurs et de l'ici. Décrire le personnage du «Chinois", par exemple, revient à exploiter la valeur fantasmatique de l'exil en accordant au regard énigmatique de l'«autre» le pouvoir de s'ancrer soi-même dans le réel, ou reconnaître, par effet de miroir, ce que l'on possède vraiment soi-même: un lieu, identifiable et différencié, étrange en sa tranquillité même.

Quelle vision a-t-on, en effet, de la Chine au Québec en 1966-1968? Au-delà des récits de Marco Polo, des pages édulcorées de Pearl Buck, des fresques historiques d'Han Suyin, des récits de Lucien Bodard ou, seulement plus tard, de Jean Pasqualini ${ }^{19}$ - mélange de clichés de luxe et de misère, de délicatesse extrême et d'extrême cruauté -, que sait-on de ce continent mystérieux?

Peu ont lu $\mathrm{Mao}^{20}$, en revanche tout le monde alors, comme Georges Dor, peut dire, paraphrasant la devise de la

mettant en scène la figure de l'étranger, Le méteque de Georges Moustaki qui selon Cécile Barthélémy - s'y définit comme Juif, Grec et Égyptien. L'engouement que suscite cette chanson en France dès sa sortie, au printemps 1969 (un an après $L \theta$ Chinois donc) rappelle celui qu'avait connu Enfants de tous pays, la chanson du Pied-Noir Enrico Macias, vers 1960 (guerre d'Algérie).

16 En termes d'universalisme, mentionnons toutefois Robert Charlebois (50 000000 d'hommes) et Raymond Lévesque (Quand les homme vivront d'amour, de 1955), d'après Bruno Roy, ibid., p. 222.

17 Autre texte de Georges Dor (information fournie par l'auteur).

18 Chanson des annóes 1970. Notons en passant combien il est paradoxal d'avoir plus de difficulté à dater telle ou telle chanson québécoise de la dernière décennie que la plupart des chansons françaises de France d'à-peu près n'importe quelle époque.

19 Jean Pasqualini et Rudolf Cheminsky, Prisonnier de Mao. Sept ans dans un camp de travail en Chine, coll. * Témoins ", Paris, Gallimard, 1975.

20 Dont les Poésies complètes seront publiées chez Parti pris, début 1972, par Gérald Godin, et lancées à... Lachine, en banlieue de Montréal, d'après les 
Province de Québec, «je m'souviens * des * p'tits Chinois de la Sainte-Enfance»; et personne ne doutera, à écouter la chanson, que cet «individu à l'allure bizarre ${ }_{*}{ }^{21}$, ce probable buandier ${ }^{22}$ de quartier «tranquille $(\mathrm{XI}, 2$ ), * qui va et qui vient* (XI, 3), ne lave le linge sale de la Sainte-Famille. À moins que, troisième hypothèse relevant encore de l'idéographie populaire, il ne soit cuisinier. Ce qu'indiquerait une lecture ludique transformant en «mets» les nombreux * mais * du texte ou, plus trivial encore, entendant dans *A petits pas soir et matin" (XI, 4) le calembour par lequel le « chinois * est rendu, en minuscule, à sa désignation commune d'instrument culinaire.

Quoi qu'il en soit, nul ne sait le Fleuve qu'il regarde. Bleu, Jaune, Rouge ou Amour: impossible de voir par la fente de ses yeux l'envers du miroir; l'image qu'il donne est celle de l'impénétrable. Figé qu'il est à coups de clichés et de préjugés les siens et les autres - prisonnier de son histoire et de son mystère, un mur d'indifférence et de mutisme s'érige autour de sa différence, qui l'isole mieux que toutes les murailles, icônes de toutes les Chines.

Ainsi, suggéré par touches successives, le personnage du "Chinois" - paraphrasons: "vieux" et courbé (I, 2), qui marche «à petits pas soir et matin * (XII, 4) - rejoint-il le collectif dans le singulier avec l'image canadienne-française traditionnelle du porteur d'eau ployant l'échine sous le fardeau. Vue du Québec, l'épopée maoïste avec « Ces enfants qui marchaient pieds nus" (VIII, 3) sollicite les résurgences biographiques ${ }^{23}$ et convoque les traces résiduelles d'une "mémoire populaire " marquée par le déracinement ${ }^{24}$, biblique ou historique. Dans la démarche particulière du personnage du "Chinois", dans son comportement même, se reflète - jeu de miroir déformant - le propre particularisme de son observateur

nombreux renseignements généreusement fournis par André Gervais. Ecrits entre 1925 et 1965, ces 38 poèmes n'ont commence à être publiés qu'en 1957 en langue originale et une première traduction française plutôt discutable parait dès 1958. Une traduction plus intéressante, accompagnée de trés nombreux commentaires, par Paul Daniéville, paraît dans le Mercure de France, en avril 1965.

21 Définition de "Chinois ", sens figuré (1845), Petit Robert.

22 Comme le laisse supposer le champ sémantique du a décor empesé " (XII, 2) dans lequel le personnage évolue.

23 Georges Dor est lui-meme descendant d'immigrés irlandais.

24 Relire a Lieux exemplaires " de Giguere (1954-1955) en fournit un exemple.

Roland Giguère, L'åge de la parole, Montréal, L'hexagone, 1965. 
et ce type de constat, de l'aveu de l'auteur, témoigne de son ignorance ${ }^{25}$ et de son désir d'ouverture à l'، autre».

\section{L'“ autre " ou l'inaccessible}

L'«autre» comme pays. «Je n'aurai jamais vu la Chine / Mais je me l'imagine" (X, 1-2): pays fabuleux, métaphore d'une poésie de l'extrême, prétexte à tous les clichés et à toutes les rêveries (je lis "ma Chine»). Ainsi, Chine traditionnelle, Chine d'opérette et de pacotille ou Chine de Mao, confondues à travers telles ou telles réminiscences poétiques et livresques, ou les bulletins d'information, ne peuvent-elles convoquer qu'une perception associative et impressionniste qui se retrouve dans les notations vagues comme «brum's et bruine" $(\mathrm{X}, 5)$.

Ce masque pourtant, calque subrepticement emprunté à la technique du pochoir ou de l'estampe, ne parvient pas à oblitérer la "ruine" d'une Chine "désenchantée / Inaccessible et tourmentée $(\mathrm{V}, 3)$ incluse à la rime ${ }^{26}$. Comme si, première à s'exercer vis-à-vis de la langue étrangère que représente la chanson, la mémoire phonique tentait en vain de gommer hors texte l'écho dysphorique de "Ces enfants qui marchaient pieds nus (VIII, 3) et ne retenait que la syllabe finale, cet "in' " perceptible à son tour comme une subtile et clandestine inclusion sonore de l'extérieur dans la réalité québécoise. Le pays, la payse ne sont-ils pas également, en deuxième sens, synonymes de compatriotes? C'est-à-dire un «autre» soi, étrange en son pays-même, comme ne cessent de le dire les poètes 27 . L'identification de l'un à l'«autre» - Québécois ou Chinois : exilé - devient alors plausible.

\section{Passer, avec le texte}

L'enfermement physique dont l'un et l'autre, enclaves vives, sont victimes est présent dans les quatre premières strophes; la chanson le désigne en la corporalité: "Vieux Chinois qui courbe l'échine» (I, 2) [...] «tu courbes le dos»

25 Conversation du 24 aout 1989.

26 Convoquant les événements de la révolution culturelle de 1966 justement?

27 D'Aragon à Giguère par exemple, depuis Malebranche: a Non, je ne vous conduirai point dans une terre étrangère, mais je vous apprendrai peut-être que vous êtes étranger dans votre propre pays. " 
(III, 1). La progression syntagmatique dépend des effets répétitifs qui miment le cheminement menu de cet homme emblème de tout un peuple. Qu'il s'agisse de répétition d'un même mot $^{28}$ ou de reprise homonymique ou homophonique ${ }^{29}$, le texte procède avec une rare économie de moyens lexicaux, en adéquation avec le mutisme forcé du sujet représenté, prisonnier d'un monde de signes et d'un mode de pensée différents.

Parallèlement en effet, toutes les rimes en «-ine" nient agrammatiquement ce «-on ", tiers exclus sans autre nom qu'un surnom: "un Robinson " ${ }^{30}$ (II, 1), entre tous reconnaissable incarnation de l'étrangeté et de l'isolement, emblème d'une insularité qui détermine l'«île» (II, 2) et l'«exil " (III, 3): "Sa maison c'est son île» (II, 2), tout aussi « inaccessible" (V, 4) au parolier que la Chine. Un lieu où, distinguo, le sujet n'habite ni ne demeure mais où il "reste", telle une trace flottante à l'abandon, faute d'ailleurs. Taxé de "régionalisme" en ce sens, ce verbe issu de l'isotopie domiciliaire à laquelle se rattache «maison» et «chez vous * (VI, 4) ${ }^{31}$ se retrouve employé comme verbe d'état à la dernière strophe où se joue la conclusion: « Mais il reste l'étranger * (XII, 1). Un simple effet de synonymie contribue donc à renforcer significativement la tonalité dysphorique du thème dont le texte se fait l'amplification. Dès la deuxième strophe en effet, tout est dit. Réalisme oblige: vie et mort se conjuguent et se confondent au présent comme deux termes équivalents d'une même entité, l'une égalant l'autre: «Il vit il meurt à sa façon » (II, 3).

Une telle intensité dans la peinture du détachement révèle la fraternité de pensée qui unit Dor à Miron et qui se manifeste intertextuellement. Tandis que l'un parle vie et mort, à la troisième personne, "Dans un décor empesé " (XII,

28 Par exemple "courbe w: I, 2 et III, 1 ; * dos ": III, 1 et 4 ; * Mao ": VIII, 5, IX, 1 et $3, X, 3$; “Chine" : sept fois et "Chinois ": neuf fois; a p'tits ": II, 4 et VII, 1 ; "petits": VII, 3 et XI, 4; * Vieux":I, 2, IV, 1, et VI, 1; « maison ": I, 3 et II, 2, disséminé en * mais ": V, $2, V I I I, 1, X, 2$ et XII, 1 et en * jamais " $X, 1$; " reste ": I, 1 et XII - en position d'ouverture et de cloture.

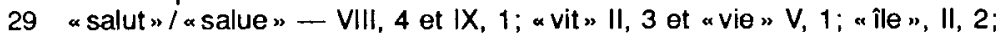
“II» II, 2; « ex-il» III, 3.

30 Allusion à Robinson Crusoë, de Daniel Defoë et, métonymiquement, allusion à l'Angleterre, ile à la fois lointaine et proche de cette autre île qu'est Montréal.

31 Rappel du slogan politique de Jean Lesage « Maîtres chez nous " (19601966)? 
2), l'autre n'affirme-t-il pas, à la première: «je vais mourir vivant dans notre empois de mort ${ }^{32}$ ? On conçoit qu'un décor aussi guindé que pouvait l'être le Québec d'alors surdétermine la figure rédemptrice du buandier: celui dont c'est le métier de prendre à son compte * l'empois » du monde, quitte à ployer sous la charge. Du déracinement il n'est pas autrement question, sinon peut-être - avec quelle précision iconographique - dans le télescopage des règnes, humain et végétal, qui inclut dans un même rythme naturel *Hommes et femm's arbres et fleurs * $(X, 4)^{33}$.

Dès lors, au-delà des apparences et des vapeurs, la "maison " de l'étranger, être rangé désigné par la régularité de ses allées et venues " soir et matin " (XI, 4), stigmatise le lieu du "Mais * récurrent de l'objection. Lieu d'une pensée différente. Généré par la réalité urbaine quotidienne, le mot "polisson " établit sous le signe du contraste - rudesse de l'un, face au «poli » des légendaires politesse et laques de l'autre - le lien entre pays neuf et civilisation ancienne et appelle, comme point de contact possible, la dimension politique. Car, toutes proportions gardées, des parallèles s'imposent. Qui conduisent à penser que tel Chinois de Longueuil pourrait être assimilé à tel Québécois d'Amérique: pacifique et passif, confit dans ses prières ou ses «chimères" (IV, 5). Pays du matin calme et Révolution tranquille se superposent en filigrane dans l'ambiguité syntaxique: "Dans ma rue bien tranquille / Un Chinois» (XI, 2/3).

Les exemples précédents le démontrent une fois de plus: tenter d'accéder à l'impénétrable et à l'inexprimable demande paradoxalement d'emprunter les voies du langage, d'en passer par les mots et le jeu des mots pour déceler les réseaux qui les unissent. Quoique l'auteur s'en défende: $\propto$ Les jeux de l'esprit nous semblent interdits " 34 et affirme que "l'échine" n'est là que pour rimer avec la "Chine ${ }^{35}$, on ne peut omettre de rappeler, au cours d'une analyse qui a pour objet * Le Chinois", à la fois passant et passeur de sens, les différentes significations du verbe «chiner * qui toutes peuvent, de près

32 Extrait du poème intitulé " La braise et l'humus ", dans le cycle de a La vie agonique ", publié pour la première fois en 1963. Gaston Miron, L'homme rapaille, p. 53.

33 Écho du titre du disque Mes ormes dans la plaine.

34 Si tu savais, p. 16.

35 Conversation du 24 août 1989. 
ou de loin, se rapporter au présent contexte. Le chansonnier n'est-il pas en effet une sorte de brocanteur à la recherche de la bonne occasion ( $1^{\text {er }}$ sens), à l'affút du sujet qui passe au quotidien, quitte à le «mettre en boîte», et à s'en moquer $\left(2^{\mathrm{e}}\right.$ sens)? (Â son insu, « la Chine [...] derrière sa mue raille ").

On sait aussi qu'initialement « chiner » signifie faire alterner - selon un procédé chinois - les couleurs sur les fils de chaîne avant de tisser, de manière à obtenir un dessin, le tissage terminé. La métaphore de la texture du texte n'est plus neuve, s'y rattachent évidemment tous les éléments scripturaires qu'évoque la Chine: du papier de luxe à l'encre indélébile. Encre noire du texte pour le buandier jaune, donc: ou l'impossibilité de «blanchir» les images de toute connotation. Force est de reconnaître sa pertinence en regard de la structure du texte: ce qu'illustre le passage du " $\mathrm{Il}$ » au « tu», ou la comparaison entre "La vie là-bas" $(\mathrm{V}, 1)$ et la vie ici, entre le Chinois et le Québécois: "Vieux Chinois tu te souviens" (VI, 1) [...] " Moi je m'souviens des p'tits Chinois» (VII, 1).

Le texte est en effet fortement connoté, de la bonhomie supérieure des pays nantis, qui fait dire, par exemple, parodie du langage des œuvres charitables que convoque la strophe IV et ce qui s'y rattache: "Quant au Chinois moi j'ai le mien» $(\mathrm{X} 1,1)$. Mais la moquerie, Georges Dor l'exerce à ses dépens jusqu'aux limites de l'insoutenable: telle "ignorance" ou naïveté enflées jusqu'à parler de "monsieur Bouddha", "tu " et «vous » mêlés tandis qu'on s'évertue à faire rimer "la Chine» avec "l'échine». Difficile aussi de ne pas soupçonner que, sous prétexte de rime avec «chez vous" (VI, 3-4) et "à vingt-cinq sous", si proche de notre "chez nous", soit entretenue la confusion homophonique entre "Sou-Tchéou" et «Dieu sait où ».

Ainsi verrait-on de même l'énoncé litanique et désormais daté des noms de lieux "De Nankin de Pékin [...]» (VI, 2-3) servir d'introït à l'évocation du pieux trafic d'âmes qui a marqué toute la génération d'acheteurs de "p'tits Chinois" (VII, 1). Ici parodie de cantiques « Le cœur plein d'allégresse " (VII, 5), le vocabulaire religieux du "salut " vient là (VIII, 4), en superposition, qualifier la Longue Marche ${ }^{36}$ et lui donne des airs de Croisade, sans que personne ne soit dupe. 
26

L'on voit bien que l'image du chef de file, «Mao en tête» (VIII, 5), est plus que descriptive: une figure obsédante digne de désorienter tout Occidental sensible aux "chinoiseries " de l'amphibologie. Mao à la tête de ses troupes, ce sont Nankin, Pékin, Canton et Sou-Tchéou (ou Hsü-Chou), respectivement villes du centre, du nord, du sud et du centre, occupées par l'Armée Rouge entre janvier et octobre 1949. "Mao en tête", c'est aussi, réponse à une Chine "Millénaire et désenchantée / Inaccessible et tourmentée» (V, 3 et 4), l'admiration inconditionnelle manifestée à l'égard de l'image du poète meneur d'hommes, présente dans la récurrence des * $M »$ du texte. Vision lointaine et floue d'une autre Chine imaginaire à laquelle s'adresse, souligné par enjambement, le semblant de dédicace finale: "pour mieux rêver / À la Chine... (XII, 4/5).

Comment une chanson peut transcender le quotidien dont elle est issue et donner une dimension universelle à un personnage anonyme: telle serait, en bref, le «message» d'un tel texte qui, par effet synecdochique ou déplacement d'un personnage, familier et inconnu, à toute une civilisation en pleine effervescence, du singulier s'élève au général. Dépassant les contingences individuelles qui font de l'homme un émigré, il hausse la singularité d'un individu à un phénomème humain et suscite en son temps l'ouverture d'une nouvelle réflexion à propos de l'exil qui trouve étrangement écho, du point de vue de l'« hôte ", dans la littérature d'aujourd'hui ${ }^{37}$.

Quant aux arrangements musicaux, ils montrent ce que telle échappée d'humanité face à l'*autre * peut parfois avoir de surprenant, de fascinant, mais aussi de dérangeant et de factice dans une société donnée, où l'intégration, par méconnaissance, sans cesse risque de sonner faux. Exclu par sa langue, son passé, son âge, sa culture, sa religion, bref: tout ce qui fait sa différence, sa singularité, l'«autre» reste, qu'on le veuille ou non, jusqu'en ses moindres gestes, attitudes ou habitudes, objet de curiosité. Mais, hors contexte, hors des lieux communs a empesés " du préjugé et au-delà des limites syntaxiques que seule peut franchir la musique, la petite phrase: "Mais il reste l'étranger" (XII, 1) signifie aussi que l'" autre » est également mot de passe et d'espoir pour d'autres modes d'expression à découvrir, indice enfin que l'« autrement » existe. Meilleur ou pire : c'est une autre chanson.

37 Pensons par exemple à la part importante du paradigme de l'immigré dans l'œuvre de Jean-Marie-Gustave Le Clézio. 
En ces termes, aux lieux mêmes du manque, la chanson, de lieu d'exclusion et de repli, devient lieu d'ouverture et prétexte à participer à une aventure humaine plus vaste. L'insignifiance des paroles, des faits qu'elle rapporte, par le biais de l'in-signifiance des mots ne fait qu'illustrer l'aveu d'ignorance qui constitue l'essentiel du dispositif paradigmatique sous-jacent. Elle stigmatise l'ignorance individuelle, indice d'une ignorance collective plus grande qui laisse l'«autre" vivre sa différence dans une indifférence tranquille et mesurée, plus cruelle que tous les supplices. En ce sens, on le voit, la plus anodine d'entre elles peut se faire porteuse de manifeste. C'est pourquoi, sans doute, il arrive qu'on s'en souvienne. Preuve que, comme l'a déjà indiqué Gaston Miron, «La poésie n'est pas la mode et pas forcément la nouveauté, mais $[\ldots .$.$] toujours actualisante et nouvelle." 38$

38 “Propositions" figurant au programme diffusé par le ministère des Affaires culturelles, à l'occasion du récital de poèmes et chansons donné par Georges Dor, les 21,22 et 23 mars 1970 à la salle Saint-Sulpice de la Bibliotheque nationale du Québec. 